\title{
Surface Resistivity of Silicone Rubber Formulations Tested in Room Ambient Conditions: The case of silicone rubber formulations with and without filler materials
}

\author{
AVITI THADEI AND ALEXANDER KYARUZI \\ Department of Electric Power Engineering \\ University of Dar es Salaam \\ Box 35131, Dar es Salaam
}

TANZANIA

aviti.thadei@udsm.ac.tz and kyaruzi@udsm.ac.tz http://www.udsm.ac.tz

\begin{abstract}
Silicone rubber formulations in the form of thin discs have been studied under room ambient conditions for their surface characteristics. The samples were silicone rubber manufactured in laboratory and those industrially manufactured. The measurements were done using an electrometer high resistance meter, applying $d c$ voltage under normal room ambient conditions. The results show that the silicone rubber samples show higher values of surface resistivity when the dc voltage was applied. Silicone rubber samples manufactured in laboratory seem to exhibit erratic behaviour unlike their corresponding silicone rubbers manufactured in industry; this could be due to manufacturing shortcomings in laboratory and the irregularities in the way the silicone rubber adhered to the concentric ring electrodes. The empirical current traversing the surface of the silicone rubbers does not decay exponentially but rather it decays as an exponential power of the energization time.
\end{abstract}

Key-Words: - Silicone Rubber, Surface Resistance, Surface Resistivity, Surface Charging.

\section{Introduction}

NCIs are increasingly being used in new power lines, which are installed in polluted environment as well as for replacements of installed ceramic and glass 
insulators during maintenance. The excellent performance of the polymeric insulators in polluted and wet conditions is attributed to the ability of the material to maintain the hydrophobicity of the surface in the presence of severe contamination and wet conditions (Gubanski et al., 2000; Zhang \& Hackam, 1998). This is because of the presence of low molecular weight (LMW) mobile fluid in the bulk of the silicone and EPDM rubbers, which diffuses to the surface and to the above contamination layer. The diffusion process occurs when the outer film of LMW is removed in the presence of dry band arcing and then washed away by the rain. Polymeric insulators thus have a good surface hydrophobicity which suppresses the onset of leakage current and increase the withstand voltage. The electrical, physical and chemical properties of the surface of the polymer insulator become critical to its continuing satisfactory and reliable service throughout the lifetime of the insulator (Zhang \& Hackam, 1998).

Other benefits of employing polymer insulators are their: resistance to weathering, excellent suppression of leakage currents, higher flashover voltage even when wet and polluted, resistance to vandalism, higher puncture strength and the famous excellent hydrophobicity.

There has been extensive research on polymeric outdoor insulation (Chen et al., 2005; Dakin, 2006; Escribe-Fillipini et al., 2001; Gorur et al., 2001; Gorur et al., 2006; Gubanski et al., 2000; Jonscher, 1999; Thornley \& Shocket, 1994; Zhang \& Hackam, 1998) to mention a few, which has resulted into better materials for outdoor insulation which can resist worst environmental conditions (Gubanski et al., 2001; Zhang \& Hackam, 1998; Gorur et al., 2006).

Despite these developments there are still many interesting phenomena worth studying which include surface resistance and resistivity, ageing of interfaces and 
change of dielectric properties of these polymer materials. Surface resistance measurements can be used as an indicator of polymer ageing in outdoor environment (Gorur et al., 2006; Gorur et al., 2001), despite the earlier problems to establish a specific value for which problems in field can occur (Gorur et al., 2001). Surface resistivity and surface resistance values are related by a simple factor of 50, which account for material geometry and electrode configurations of the electrometer probe, used (Keithley, 2003a, 2003b). These means that the values obtained in this study are for those silicone rubber when in room ambient conditions using concentric ring electrodes and energized by a $400 \mathrm{Vdc}$.

\section{Sample Preparation}

Test samples consisted of silicone rubbers, some of which were manufactured in laboratory and some were industrially manufactured. First type of sample of silicone rubber was prepared from a commercially available silicone elastomer. These silicone rubber samples were in two groups; first group (sample S1 and sample S2) were cured at an oven temperature of $120{ }^{\circ} \mathrm{C}$ for 120 minutes and second group (sample S481 and sample S482) were cured at room temperature $\left(22{ }^{\circ} \mathrm{C}-25{ }^{\circ} \mathrm{C}\right)$ for 48 hours, see Table 1 . Second type of sample of silicone rubber was a HTV silicone rubber (sample SF1 and sample SF2), and third type of sample of silicone rubber was a HTV silicone rubber (PS) used for insulator shed manufacturing delivered to the laboratory by the manufacturers. These variations gave silicone rubber samples with different characteristics, i.e., silicone rubber with no additives (S1, S2, S481 and S482), silicone rubber with additives (SF1, SF2 and PS) and different curing times and temperatures (see Table 1). 
Table 1: Sample Manufacturing Parameters

\begin{tabular}{|c|c|c|c|}
\hline Name & $\begin{array}{l}1^{\text {st }} \text { Curing time } \\
\text { and temperature }\end{array}$ & $\begin{array}{l}2^{\text {nd }} \text { Curing time } \\
\text { and temperature }\end{array}$ & Thickness \\
\hline S1 & \multicolumn{2}{|c|}{120 minutes at $120^{\circ} \mathrm{C}$} & $1.0 \mathrm{~mm}$ \\
\hline S2 & \multicolumn{2}{|c|}{120 minutes at $120^{\circ} \mathrm{C}$} & $1.0 \mathrm{~mm}$ \\
\hline S481 & \multicolumn{2}{|c|}{48 hours at $22-25^{\circ} \mathrm{C}$} & $1.0 \mathrm{~mm}$ \\
\hline S482 & \multicolumn{2}{|c|}{48 hours at $22-25^{\circ} \mathrm{C}$} & $1.0 \mathrm{~mm}$ \\
\hline SF1 & \multicolumn{2}{|c|}{ HTV rubber } & $3.0 \mathrm{~mm}$ \\
\hline SF2 & \multirow{2}{*}{\multicolumn{2}{|c|}{$\begin{array}{l}\text { HTV rubber } \\
\text { HTV rubber }\end{array}$}} & $3.0 \mathrm{~mm}$ \\
\hline PS & & & $2.0 \mathrm{~mm}$ \\
\hline
\end{tabular}

For those silicone rubber samples manufactured in laboratory had thickness of $1 \mathrm{~mm}$ and those delivered by manufacturers had $2 \mathrm{~mm}$ and $3 \mathrm{~mm}$ as shown in Table 1 . In addition, it must be noted the naming of the silicone rubbers has some letters and some numbers. The name contains information such as the type and the curing time. For example, sample S481 means it is a first sample made from an elastomer, which has been cured in laboratory at a room temperature of $22-25{ }^{\circ} \mathrm{C}$ in the duration of 48 hours.

\section{Experimental}

Surface resistivity measurements were carried out in room ambient conditions of 22 ${ }^{\circ} \mathrm{C}-25{ }^{\circ} \mathrm{C}$ and relative humidity of $32 \%-40 \%$. Electrometer high resistance meter (Keithley model 6517A) supplied the test dc voltage of $400 \mathrm{~V}$ and sample was placed in a resistivity test fixture (Keithley model 8009) with concentric ring electrodes. The test voltage was applied for about 50 minutes. The Electrometer was used to display the instantaneous resistivity values but it was also connected to a desktop computer through a GPIB bus, which allowed data to be collected in a computer. The data sampling was done by using LABVIEW $^{\mathrm{TM}}$ software collecting data at an interval of 90 ms. Data analysis was done with the help of a MATLAB ${ }^{\mathrm{TM}}$ software. 


\subsection{Data Analysis}

Instantaneous surface resistivity values were having a big spread. Due to this it was deemed important to use linear regression techniques (Matlab, 2007) to smooth the data to obtain smooth curves when plotting. Each smoothed value was determined by neighboring data defined within a span of $15 \%$. The process employed robust functions to make the resulting values resistant to outliers by using $1^{\text {st }}$ degree polynomial function of linear least squares regression.

\section{Results}

Results from the experiments are presented in Fig.1 for the time variations of surface resistivity in first 50 minutes.

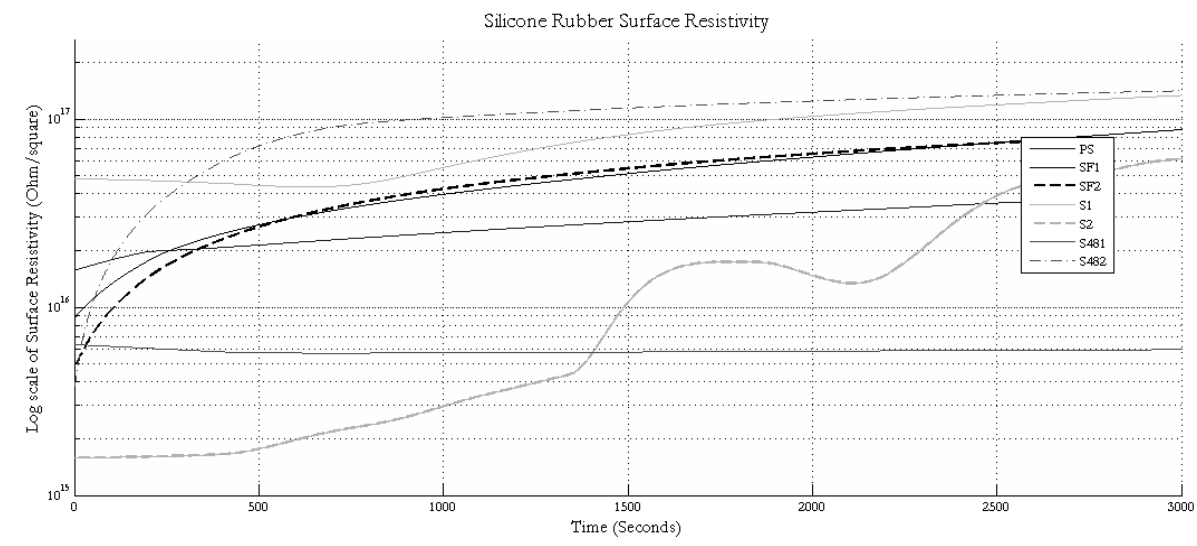

Fig.1: Silicone rubber samples surface resistivity variation with time for the first 50 minutes. The conditions of the test were room temperature, pressure and humidity i.e. temperature $=22{ }^{\circ} \mathrm{C}-25^{\circ} \mathrm{C}$, humidity $=40 \%$.

It can be seen that surface resistivity values are given in log scale to accommodate very small values and very high values i.e., $0 \sim 10^{17}(\mathrm{Ohm} / \mathrm{square})$ in horizontal axis of Fig.1. It is worth explaining here that the units given are not the SI units of surface resistivity rather, are units which are used for convenience of differentiating surface resistivity from surface resistance (Keithley, 2003a, 2003b; 
Maryniak et al., 2003) since, both have Ohm as their standard unit according to dimensional analysis.

\subsection{Discussion of the Results}

In the plots of Fig.1 silicone rubber samples resistivity values for the duration of 3000 seconds (50 minutes) is presented. Silicone rubber shows varying values of surface resistivity after the first $2-3$ minutes $(120-180$ seconds $)$ as seen in Fig.1. Samples S1 and sample S2 shows very different values from each other, since S1 shows a stable behavior but S2 shows an erratic behavior. It is seen that sample S2 had a lot of spikes even after smoothing the data. This could be due to the reason of interference since during that time there was transformer measurements being undertaken in the same laboratory.

Samples S481 and S482 both shows stable but different values. S481 shows pretty stabilized values for the increasing time, whilst S482 shows a higher value but slowly increasing after 1000 seconds. Samples SF1 and sample SF2 both exhibit same curves. Sample PS exhibited behaviour similar to samples SF1 and SF2 although at a slightly lower values of surface resistivity.

The main reason for the differing values exhibited by samples S1, S2, S481 and S482 could be a contact problem of the electrodes, since the manufacturing process could not guarantee perfect smooth surface finish, and the rubber was very soft such that it could adhere to one part of the electrode and leave a gap on another part, for which case industrially made rubbers (SF1, SF2 and PS) showed some consistent behaviour with one another due to their smooth surface finish and hardness. Another thought reason could be caused by the electrodes affecting charge formation on the materials (Chen et al., 2005) and other reasons as explained elsewhere (Escribe-Fillipini et al., 2001). 


\subsection{Comparison with other Surface Resistance Measurements Results}

From measurements it was possible to estimate the average value of surface resistance of the silicone rubber (see Table 1) for the first five minutes. Comparing results of this study with the resistance results of Gorur et al. (2001) measurements on full polymer insulators, the lowest values of surface resistance for the uncontaminated insulators are slightly lower than the values obtained in this study. This difference is due to the fact that for those measurements in Gorur et al., a stress of $15 \mathrm{kV}$ was used on full insulators while in this study only $400 \mathrm{~V}$ was used for disc samples.

\section{Conclusion}

One thing noted was that the samples had a tendency of retaining some charges inside them which could be a little explained elsewhere (Chen et al., 2005), although in that paper emphasis was done on ac stress aging; (Dakin, 2006; Jonscher, 1999) which explain about the charging of dielectrics. After energizing the material for roughly 5 minutes, if the energization process was interrupted and resumed later, the material quickly reached the level of the previous value. This tendency implies polarization of the samples by the dc voltage and higher relaxation times of the samples (Dakin, 2006; Jonscher, 1999).

If the energization was done for longer than 50 minutes the resistivity values continued to increase up to the point that the data obtained was not useful since the values became so high that the instrument generated overflow (Keithley, 2003a, 2003b). This has to be explained by the fact that the current measured becomes so low to be measured by our instrument (Keithley, 2003a, 2003b; Escribe-Fillipini et al., 2001).

From these results depicted by Fig. 1, it becomes obvious that the complex nature of the dielectrics studied; the empirical current that traverses the surface of the insulating 
materials does not decay exponentially but rather as an exponential power of the time as by equation (1) from (Dakin, 2006):

$$
\mathrm{I}=\mathrm{A}_{\mathrm{o}} \mathrm{t}^{-\mathrm{n}}
$$

Until now it is still very difficult to establish the lifetimes of polymeric insulators in field (Gubanski et al., 2000; Zhang \& Hackam, 1998) but the ongoing researches on the surface behaviour are promising. Consequently, as done by Filippini et al. (2001); Gorur et al. (2001); and Gorur et al. (2006): square wave is used to characterize the surface resistance of fully polymeric insulators. It can be stressed out that the study of surface resistance and surface resistivity is a promising research area for both Materials Scientists and Electrical Engineers.

\section{Acknowledgements}

The author acknowledges the support of Sida/SAREC Capacity Building Project of UDSM for funding this research. In addition, S. M. Gubanski, J. Andersson and all staff of Elteknik of Chalmers are acknowledged for availing laboratory space, materials and instruments to conduct the measurements.

\section{References:}

[1] S. M. Gubanski, M. A. R. M. Fernando, S. J. Pietr, J. Matula, A. Kyaruzi, Effects of Biological Contamination on Insulator Performance, Proceedings of the $6^{\text {th }}$ International Conference on Properties and Applications of Dielectric Materials, Xi'an, China, 2000. 
[2] H. Zhang, R. Hackam, Surface Resistance and Hydrophobicity of HTV Silicone Rubber in Presence of Salt-fog, IEEE International Symposium on Electrical Insulation, Arlington, Virginia, USA, 1998, pp. 355-359.

[3] R. S. Gorur, H. M. Schneider, J. Cartwright, Y. Beausajour, K. Kondo, S. M. Gubanski, R. Hartings, M. Shah, J. McBride, C. de Tourreil, Z. Szilagyi, Surface Resistance Measurements on Nonceramic Insulators, A Task Force Report of the IEEE Working Group on Insulator Contamination, IEEE Transactions on Power Delivery, Vol.16, NO.4, 2001, pp. 801-805.

[4] Keithley Model 6517A User's Manual, Rev. D, 2003.

[5] Keithley Model 8009 User's Manual, 2003.

[6] W. A. Maryniak, T. Uehara, M. A. Noras, Surface Resistivity and Surface Resistance Measurements Using Concentric Ring Probe Technique, Trek Application Note, NO.1005, 2003.

[7] A. K. Jonscher, Dielectric Relaxation in Solids, Review Article, Journal of Physics, 1999, pp. R57-R70.

[8] MATLAB Help File @ 2005.

[9] D. Thornley, A. Shocket, 25 Years Experience of Outdoor Polymeric Insulation, IEEE Transactions, 1994, pp. R67-R72.

[10] G. Chen, M. Fu, X. Z. Liu, L. S. Zhong, AC Aging and Space-Charge Characteristics in Low Density Polyethylene Polymeric Insulation, Journal of Applied Physics 97, 2005.

[11] C. Escribe-Filippini, R. Tobazêon, J. C. Filippini, Conduction Characterization of Polymer Films Using the Alternate Square Wave Methods, $20017^{\text {th }}$ International Conference on Solid Dielectrics, Eindhoven, the Netherlands, 2001, pp. 385-318. 
[12] Gorur, R. S., Crane, J., Adams, T., Gurney, J., Duxbury, J. \& Venkataraman, S. (2006b). Prediction of Flashover Voltage Using Low Voltage Surface Resistance Measurement. Final Project Report, Power Systems Engineering Research Center (PSERC), PSERC Publication 06 - 42.

[13] Dakin, T. W. (2006). Conduction and Polarization Mechanisms and Trends in Dielectrics. DEIS Feature Article, September - October 2006, Vol. 22, No. 5. 